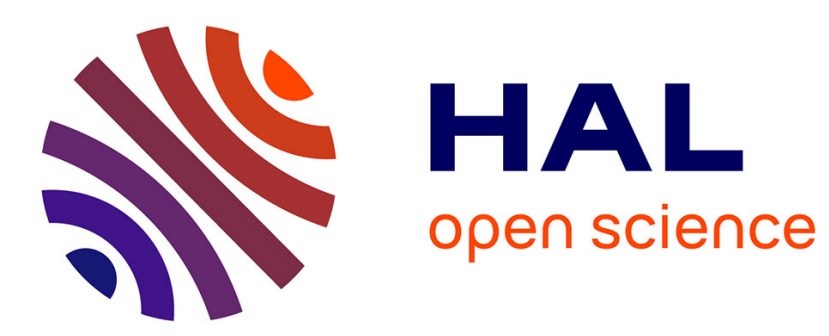

\title{
On the elastic fields produced by nonuniformly moving dislocations: a revisit
}

Markus Lazar

\section{To cite this version:}

Markus Lazar. On the elastic fields produced by nonuniformly moving dislocations: a revisit. Philosophical Magazine, 2011, pp.1. 10.1080/14786435.2011.579584 . hal-00712752

HAL Id: hal-00712752

https://hal.science/hal-00712752

Submitted on 28 Jun 2012

HAL is a multi-disciplinary open access archive for the deposit and dissemination of scientific research documents, whether they are published or not. The documents may come from teaching and research institutions in France or abroad, or from public or private research centers.
L'archive ouverte pluridisciplinaire HAL, est destinée au dépôt et à la diffusion de documents scientifiques de niveau recherche, publiés ou non, émanant des établissements d'enseignement et de recherche français ou étrangers, des laboratoires publics ou privés. 


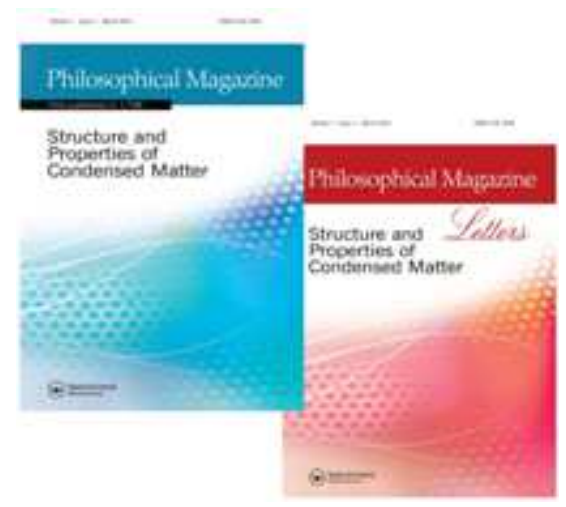

\section{On the elastic fields produced by nonuniformly moving dislocations: a revisit}

\begin{tabular}{|r|l|}
\hline Journal: & Philosophical Magazine \& Philosophical Magazine Letters \\
\hline Manuscript ID: & TPHM-10-Nov-0485.R1 \\
\hline Journal Selection: & Philosophical Magazine \\
\hline Date Submitted by the \\
Author: & 31 -Mar-2011 \\
\hline Complete List of Authors: & $\begin{array}{l}\text { Lazar, Markus; Institute of Condensed Matter Physics, Darmstadt } \\
\text { University of Technology }\end{array}$ \\
\hline Keywords: & dislocation dynamics, elastic waves, elasticity \\
\hline Keywords (user supplied): & nonuniform motion, elastodynamics \\
\hline &
\end{tabular}

\section{SCHOLARONE ${ }^{\text {MN }}$ Manuscripts}




\title{
On the elastic fields produced by nonuniformly moving dislocations: a revisit
}

\author{
Markus Lazar a,b,* \\ ${ }^{a}$ Heisenberg Research Group, \\ Department of Physics, \\ Darmstadt University of Technology, \\ Hochschulstr. 6, \\ D-64289 Darmstadt, Germany \\ b Department of Physics, \\ Michigan Technological University, \\ Houghton, MI 49931, USA
}

March 31, 2011

\begin{abstract}
We investigate the nonuniform motion of straight dislocations in infinite media using the theory of incompatible elastodynamics. The equations of motion are derived for nonuniformly moving screw dislocation, gliding edge and climbing edge dislocations. The exact closed-form solutions of the elastic fields are calculated. The fields of the elastic velocity and elastic distortion surrounding the arbitrarily moving dislocations are given explicitely in the form of integral representations free of non-integrable singularities. The elastic fields describe the response in the form of nonuniformly moving elastic waves caused by the dislocation motion.
\end{abstract}

Keywords: dislocation dynamics; nonuniform motion; elastodynamics.

\section{Introduction}

The investigation of the nonuniform motion of dislocations has attracted the interest of researchers in different fields such as material science, continuum mechanics, seismology and earthquake engineering (see, e.g., [1, 2]). Dislocation movements can be considered

${ }^{*}$ E-mail address: lazar@fkp.tu-darmstadt.de (M. Lazar). 
as one of the dominant wavesources. It should be emphasized that the problem considered in this paper is important for the understanding of several physical phenomena such as acoustic emission signals, dislocation avalanches and elastic waves in geophysics (see, e.g., [3]). The motion of dislocations is usually investigated in the framework of incompatible elastodynamics where the dislocation density and dislocation current tensors are given as source terms of the elastic fields (see, e.g., $[4,5,6,7,8,9]$ ). It is known that in continuum elastodynamics the behaviour of a dislocation is somehow particular, because at any time the fields are determined not only by the instantaneous values of the velocity (or higher derivatives of the position with respect to time), but also by the values in the past $[10,11]$. As Eshelby [10] succinctly put it: 'The dislocation is haunted by its past'. This fact is based on the physical property that Huygens' principle is not valid in two dimensions (see, e.g., [12]).

Using an electromagnetic analogy, Eshelby [11] derived simple solutions of inhomogeneous wave equations for the velocity and elastic distortion fields in terms of 'stress functions' of a nonuniformly moving screw dislocation. The solution is given in the form of time integrals. Eshelby [11] pointed out that the displacement field is of less physical importance than the elastic fields of a moving screw dislocation. The direct solution of the elastic fields given in [11] involves much simpler integrals than the integrals occurring for the displacement of a nonuniformly moving screw dislocation given by Eshelby [10] and Nabarro [13]. Later, Kiusalaas and Mura [14] found solutions in terms of 'stress (or potential) functions' for the velocity and elastic distortion fields for a gliding edge dislocation moving nonuniformly (see also [1,9]). Weertman and Weertman [1] pointed out that the problem of the climbing edge dislocation which moves at an arbitrary velocity has not been considered in any published paper. Furthermore, to the knowledge of the author of this paper a similar solution for a nonuniformly climbing edge dislocation has been still lacking in the literature. Thus, the case of a climbing edge dislocation is an outstanding problem. In addition, a systematic presentation of the elastic fields produced by the nonuniform motion of screw and edge dislocations is missing is the literature so far. Even in standard books of dislocation theory $[6,15,16,17,18]$ this topic is not covered. This is surprising because the nonuniform motion of dislocations is a fundamental problem in dislocation theory like the Liénard-Wiechert potentials in electromagnetodynamics. The reason lies in the complexity of the nonuniform motion of dislocations. In the present paper, we give a clear derivation for the elastic fields of nonuniformly moving dislocations using the 'improved' Mura equations. We investigate a screw dislocation, a gliding edge dislocation and a climbing edge dislocation. For the first time, we give the elastic fields of a nonuniformly climbing edge dislocation.

Markenscoff [19] (see also [21]) pointed out that some care is necessary in the calculation of the elastic fields produced by nonuniformly moving dislocations in order to avoid non-integrable singularities. Markenscoff [19] proved that the general expressions for the velocity and elastic distortion fields of dislocations given by Mura $[4,5]$ are not free of non-integrable singularities. The reason is that the integration and differentiation cannot be changed in these cases. As a consequence the differentiation has to be outside the integral in order to obtain correct results. In the application to screw and gliding edge dislocations, Kiusalaas and Mura [14, 22] used the correct interpretation with a differentiation outside the integrals. 
The paper is organized as follows: in Section 2, we present the framework of incompatible elastodynamics and we give the equations of motion, Mura's integral expressions for the elastic velocity and elastic distortion fields as well as the two-dimensional Green tensors. The elastic fields of nonuniformly moving screw dislocation, gliding edge and climbing edge dislocations are presented in Sections 3, 4 and 5, respectively. The static limit of the elastic fields of the nonuniformly moving dislocations is given in Section 6 .

\section{Equations of motion of dislocations}

In this section, we give the equations of motion for dislocations in the framework of incompatible elastodynamics (see, e.g., [4, 5, 6, 23]). In elasticity without external forces, the equilibrium condition reads ${ }^{1}$

$$
\dot{p}_{i}=\sigma_{i j, j}
$$

where $\boldsymbol{p}$ and $\boldsymbol{\sigma}$ are the linear momentum vector and the force stress tensor, respectively. In the incompatible linear elasticity, the momentum vector $\boldsymbol{p}$ and the stress tensor $\boldsymbol{\sigma}$ can be expressed in terms of the incompatible elastic velocity vector $\boldsymbol{v}$ and the incompatible elastic distortion tensor $\boldsymbol{\beta}$ by means of the following constitutive relations

$$
\begin{aligned}
p_{i} & =\rho v_{i}, \\
\sigma_{i j} & =C_{i j k l} \beta_{k l},
\end{aligned}
$$

where $\rho$ is the mass density and $C_{i j k l}$ is the tensor of elastic moduli. The tensor $C_{i j k l}$ possesses the following symmetry properties

$$
C_{i j k l}=C_{j i k l}=C_{i j l k}=C_{k l i j} .
$$

If we substitute the constitutive relations (2) and (3) in Eq. (1), we obtain the equilibrium condition expressed in terms of the elastic fields $\boldsymbol{v}$ and $\boldsymbol{\beta}$

$$
\rho \dot{v}_{i}=C_{i j k l} \beta_{k l, j}
$$

The presence of dislocations makes the elastic fields incompatible which means that they are not anymore simple gradients or time derivatives of a displacement vector $\boldsymbol{u}$. In the incompatible elasticity, the elastic fields can be given in terms of the displacement field $\boldsymbol{u}$, the plastic distortion $\boldsymbol{\beta}^{\mathrm{P}}$ and the plastic velocity $\boldsymbol{v}^{\mathrm{P}}[24,25]$

$$
\begin{aligned}
\beta_{i j} & =u_{i, j}-\beta_{i j}^{\mathrm{P}}, \\
v_{i} & =\dot{u}_{i}-v_{i}^{\mathrm{P}} .
\end{aligned}
$$

The plastic distortion $\boldsymbol{\beta}^{\mathrm{P}}$ is a well-known quantity in the dislocation theory. The plastic velocity $\boldsymbol{v}^{\mathrm{P}}$ was introduced by Kossecka [24] and its existence has not been widely recognized. However, for elasto-plastic deformations caused by dislocations a part of the

\footnotetext{
${ }^{1}$ We use the usual notation $\beta_{i j, k}:=\partial_{k} \beta_{i j}$ and $\dot{\beta}_{i j}:=\partial_{t} \beta_{i j}$.
} 
motion may be of plastic nature [26]. In general, the plastic fields may be the discontinuous parts of the total fields. Nowadays, the plastic fields can be better understood as gauge fields in the framework of dislocation gauge theory [27, 28]. In contrast to the displacement field and the plastic fields, the elastic fields are physical state quantities of dislocations. For that reason, we deal only with the calculation of the elastic fields in this paper.

The dislocation density and dislocation current tensors are defined by

$$
\begin{aligned}
T_{i j k} & =\beta_{i k, j}-\beta_{i j, k}, \\
I_{i j} & =\dot{\beta}_{i j}-v_{i, j},
\end{aligned}
$$

or they read in terms of the plastic fields

$$
\begin{aligned}
T_{i j k} & =-\beta_{i k, j}^{\mathrm{P}}+\beta_{i j, k}^{\mathrm{P}}, \\
I_{i j} & =-\dot{\beta}_{i j}^{\mathrm{P}}+v_{i, j}^{\mathrm{P}}
\end{aligned}
$$

and $T_{i j k}=-T_{i k j}$. Moreover, they fulfill the Bianchi identities

$$
\begin{aligned}
\epsilon_{j k l} T_{i j k, l} & =0, \\
\dot{T}_{i j k}+I_{i j, k}-I_{i k, j} & =0,
\end{aligned}
$$

which are 'conservation' laws. Here $\epsilon_{j k l}$ denotes the Levi-Civita tensor. Eq. (12) states that dislocations cannot end inside the medium and Eq. (13) means that the time evolution of the dislocation density tensor $\boldsymbol{T}$ is determined by the 'curl' of the dislocation current tensor $\boldsymbol{I}$. For straight dislocations we have also the relation

$$
I_{i j}=V_{k} T_{i j k}
$$

where $V_{k}$ denotes the velocity of the moving dislocation.

By differentiating Eq. (5) with respect to $x_{m}$ and substituting Eqs. (8) and (9) into it, we find the dynamic elasticity-theory equation for the determination of the incompatible elastic distortion tensor $\boldsymbol{\beta}$

$$
\rho \ddot{\beta}_{i m}-C_{i j k l} \beta_{k m, j l}=C_{i j k l} T_{k m l, j}+\rho \dot{I}_{i m} .
$$

This is the equation of motion for $\boldsymbol{\beta}$ where the dislocation density and the dislocation current tensors are the sources. Eq. (15) is an anisotropic tensorial Navier equation for $\boldsymbol{\beta}$.

Similarly, performing the differentiation of Eq. (5) with respect to time and using Eq. (9), we obtain the dynamic elasticity-theory equation for the determination of the incompatible elastic velocity vector $\boldsymbol{v}$

$$
\rho \ddot{v}_{i}-C_{i j k l} v_{k, j l}=C_{i j k l} I_{k l, j} .
$$

Eq. (16) is the equation of motion for $\boldsymbol{v}$ with the dislocation current tensor as source term. Eq. (16) is an anisotropic vectorial Navier equation for $\boldsymbol{v}$. 
The solutions of Eqs. (15) and (16) can be represented as convolution integrals [4, 5, $24,29]$. In an unbounded medium and under the assumption of zero initial conditions, which means that $\boldsymbol{\beta}\left(\boldsymbol{r}, t_{0}\right)$ and $\boldsymbol{v}\left(\boldsymbol{r}, t_{0}\right)$ and their first time derivative are zero for $t_{0} \rightarrow-\infty$, the solutions of $\boldsymbol{\beta}$ and $\boldsymbol{v}$ are given in terms of the Green tensor. Therefore, the convolution integrals for the elastic fields, which are free of non-integrable singularities, are given by (see also [19])

$$
\begin{aligned}
\beta_{i m}(\boldsymbol{r}, t)= & \partial_{k} \int_{-\infty}^{t} \int_{-\infty}^{\infty} C_{j k l n} G_{i j}\left(\boldsymbol{r}-\boldsymbol{r}^{\prime}, t-t^{\prime}\right) T_{l m n}\left(\boldsymbol{r}^{\prime}, t^{\prime}\right) \mathrm{d} \boldsymbol{r}^{\prime} \mathrm{d} t^{\prime} \\
& +\partial_{t} \int_{-\infty}^{t} \int_{-\infty}^{\infty} \rho G_{i j}\left(\boldsymbol{r}-\boldsymbol{r}^{\prime}, t-t^{\prime}\right) I_{j m}\left(\boldsymbol{r}^{\prime}, t^{\prime}\right) \mathrm{d} \boldsymbol{r}^{\prime} \mathrm{d} t^{\prime}
\end{aligned}
$$

and

$$
v_{i}(\boldsymbol{r}, t)=\partial_{k} \int_{-\infty}^{t} \int_{-\infty}^{\infty} C_{j k l m} G_{i j}\left(\boldsymbol{r}-\boldsymbol{r}^{\prime}, t-t^{\prime}\right) I_{l m}\left(\boldsymbol{r}^{\prime}, t^{\prime}\right) \mathrm{d} \boldsymbol{r}^{\prime} \mathrm{d} t^{\prime}
$$

Here, $G_{i j}$ is the dynamic Green tensor of the anisotropic Navier equation defined by

$$
\left[\delta_{i k} \rho \partial_{t t}-C_{i j k l} \partial_{j} \partial_{l}\right] G_{k m}=\delta_{i m} \delta(t) \delta(\boldsymbol{r})
$$

where $\delta($.$) denotes the Dirac delta function and G_{i j}=G_{j i}$. The expressions for the elastic fields given in Eqs. (17) and (18) differ from the expressions given by Mura [4, 5, 6], Kossecka [24] and Teodosiu [29] in that the differentiation is outside the integral, as required to obtain correct results for dislocations. In the expressions given by Mura $[4,5,6]$ the differentiation of the Green tensor leads to non-integrable singularities as pointed out by Markenscoff [19]. We would like to mention that Mura [4, 5], Bross [7], Teodosiu [29] and Markenscoff [19] assumed $\boldsymbol{v}^{\mathrm{P}}=0$, so that they made no distinction between $\boldsymbol{v}$ and $\dot{\boldsymbol{u}}$.

For an isotropic material the tensor of elastic moduli reduces to

$$
C_{i j k l}=\lambda \delta_{i j} \delta_{k l}+\mu\left(\delta_{i k} \delta_{j l}+\delta_{i l} \delta_{j k}\right)
$$

where $\lambda$ and $\mu$ being the Lamé constants. Substituting Eq. (20) in Eq. (19), we obtain the isotropic Navier equation for the dynamic Green tensor

$$
\left[\delta_{i k} \rho \partial_{t t}-\delta_{i k} \mu \Delta-(\lambda+\mu) \partial_{i} \partial_{k}\right] G_{k m}=\delta_{i m} \delta(t) \delta(\boldsymbol{r})
$$

where $\Delta$ denotes the Laplacian. For the calculation of the elastic fields of straight dislocations, the problem is two-dimensional (plane strain and anti-plane strain), consequently we need the two-dimensional Green tensors. If the material is infinitely extended, the dynamic Green tensor of plane strain reads [30, 31]

$$
\begin{aligned}
G_{i k}=\frac{1}{2 \pi \rho}\{ & \frac{x_{i} x_{k}}{r^{4}}\left(\frac{\left[2 t^{2}-r^{2} / c_{\mathrm{L}}^{2}\right]}{\sqrt{t^{2}-r^{2} / c_{\mathrm{L}}^{2}}} H\left(t-r / c_{\mathrm{L}}\right)-\frac{\left[2 t^{2}-r^{2} / c_{\mathrm{T}}^{2}\right]}{\sqrt{t^{2}-r^{2} / c_{\mathrm{T}}^{2}}} H\left(t-r / c_{\mathrm{T}}\right)\right) \\
& \left.-\frac{\delta_{i k}}{r^{2}}\left(\sqrt{t^{2}-r^{2} / c_{\mathrm{L}}^{2}} H\left(t-r / c_{\mathrm{L}}\right)-\frac{t^{2}}{\sqrt{t^{2}-r^{2} / c_{\mathrm{T}}^{2}}} H\left(t-r / c_{\mathrm{T}}\right)\right)\right\}
\end{aligned}
$$


and the dynamic Green tensor of anti-plane strain is given by

$$
G_{z z}=\frac{1}{2 \pi \rho c_{\mathrm{T}}^{2}} \frac{H\left(t-r / c_{\mathrm{T}}\right)}{\sqrt{t^{2}-r^{2} / c_{\mathrm{T}}^{2}}}
$$

where $H($.$) denotes the Heaviside step function and r=\sqrt{x^{2}+y^{2}}$. The constants $c_{\mathrm{L}}$ and $c_{\mathrm{T}}$ denote the velocities of the longitudinal and transversal elastic waves (sometimes called $\mathrm{P}$ and S-waves). The sound velocities can be given in terms of the Lamé constants $\left(c_{\mathrm{T}}<c_{\mathrm{L}}\right)$

$$
c_{\mathrm{L}}=\sqrt{\frac{2 \mu+\lambda}{\rho}}, \quad c_{\mathrm{T}}=\sqrt{\frac{\mu}{\rho}} .
$$

Let us now discuss some important properties of the Green tensors of plane strain and anti-plane strain. The Green tensor of anti-plane strain $G_{z z}$ diverges to $+\infty$ at $r \rightarrow c_{\mathrm{T}} t^{-}$ and converges to $1 /\left(2 \pi \rho c_{\mathrm{T}}^{2}|t|\right)$ at $r \rightarrow 0$ (see Fig. 1a). On the other hand, the Green tensor of plane strain (22) consists of two characteristic pieces. Note that the expression

$$
\frac{1}{r^{2}}\left(\frac{\left[2 t^{2}-r^{2} / c_{\mathrm{L}}^{2}\right]}{\sqrt{t^{2}-r^{2} / c_{\mathrm{L}}^{2}}} H\left(t-r / c_{\mathrm{L}}\right)-\frac{\left[2 t^{2}-r^{2} / c_{\mathrm{T}}^{2}\right]}{\sqrt{t^{2}-r^{2} / c_{\mathrm{T}}^{2}}} H\left(t-r / c_{\mathrm{T}}\right)\right)
$$

diverges to $+\infty$ at $r \rightarrow c_{\mathrm{L}} t^{-}$and it converges to 0 at $r \rightarrow 0$. At $r=c_{\mathrm{T}} t$ it possesses a jump: it diverges to $-\infty$ at $r \rightarrow c_{\mathrm{T}} t^{-}$and converges to a finite-limit value of $\left(2 c_{\mathrm{L}}^{2}-\right.$ $\left.c_{\mathrm{T}}^{2}\right) /\left(c_{\mathrm{L}}^{2} c_{\mathrm{T}}^{2} \sqrt{\left(c_{\mathrm{L}}^{2}-c_{\mathrm{T}}^{2}\right) / c_{\mathrm{L}}^{2}}|t|\right)$ as $r \rightarrow c_{\mathrm{T}} t^{+}$(see Fig. 1b). Thus, discontinuities exist for both wavefronts. The piece

$$
\frac{1}{r^{2}}\left(\frac{t^{2}}{\sqrt{t^{2}-r^{2} / c_{\mathrm{T}}^{2}}} H\left(t-r / c_{\mathrm{T}}\right)-\sqrt{t^{2}-r^{2} / c_{\mathrm{L}}^{2}} H\left(t-r / c_{\mathrm{L}}\right)\right)
$$

converges to 0 at $r \rightarrow c_{\mathrm{L}} t^{-}$and converges to a finite-limit value of $\left(c_{\mathrm{L}}^{2}+c_{\mathrm{T}}^{2}\right) /\left(2 c_{\mathrm{L}}^{2} c_{\mathrm{T}}^{2}|t|\right)$ at $r \rightarrow 0$. At $r=c_{\mathrm{T}} t$ it possesses a jump: it diverges to $+\infty$ at $r \rightarrow c_{\mathrm{T}} t^{-}$and converges to a finite-limit value of $-1 /\left(c_{\mathrm{T}}^{2}|t|\right) \sqrt{\left(c_{\mathrm{L}}^{2}-c_{\mathrm{T}}^{2}\right) / c_{\mathrm{L}}^{2}}$ as $r \rightarrow c_{\mathrm{T}} t^{+}$(see Fig. 1c). Therefore, a discontinuity exists for the wavefront at $r=c_{\mathrm{T}} t$. The combination of the two pieces gives a finite value at $r=c_{\mathrm{T}} t$ and at $r=c_{\mathrm{L}} t$ it diverges to $+\infty$ (see Fig. 1d). A discontinuity exists only for the wavefront at $r=c_{\mathrm{L}} t$. Thus, in general, at the wavefronts $r=c_{\mathrm{T}} t$ and $r=c_{\mathrm{L}} t$ the Green tensors possess singularities and discontinuities and for small $r$ they possess a long tail of the typical two-dimensional pulse. Physically, there is afterglow implying that Huygens' principle is not valid in two dimensions. Although two waves with the velocities $c_{\mathrm{L}}$ and $c_{\mathrm{T}}$ exist, there is never complete separation between the two. These properties of the Green tensors will influence also the properties of the 'potential functions' (retarded potentials) and the elastic fields of dislocations in that way that they are influenced or haunted by their past. Thus, the expression (22) shows that the disturbances are propagated with the velocities $c_{\mathrm{T}}$ and $c_{\mathrm{L}}$. At the wavefronts $r=c_{\mathrm{T}} t$ and $r=c_{\mathrm{L}} t$ the Green tensor (22) shows algebraic singularities. In Fig. 1 the Green tensors $G_{z z}, G_{x y}$ for $\varphi=\pi / 4, G_{x x}$ for $\varphi=\pi / 2$ and $G_{x x}$ for $\varphi=0$ are plotted over $r$ for fixed time $t$.

It should be noted that for the calculation of the elastic fields produced by dislocation loops and curved dislocations the three-dimensional Green tensor $G_{i j}$ is needed (see also $[20,21])$. 


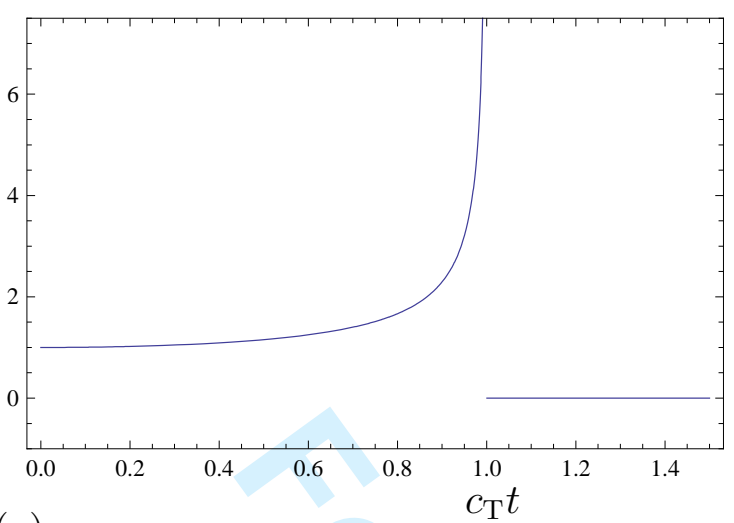

(a)

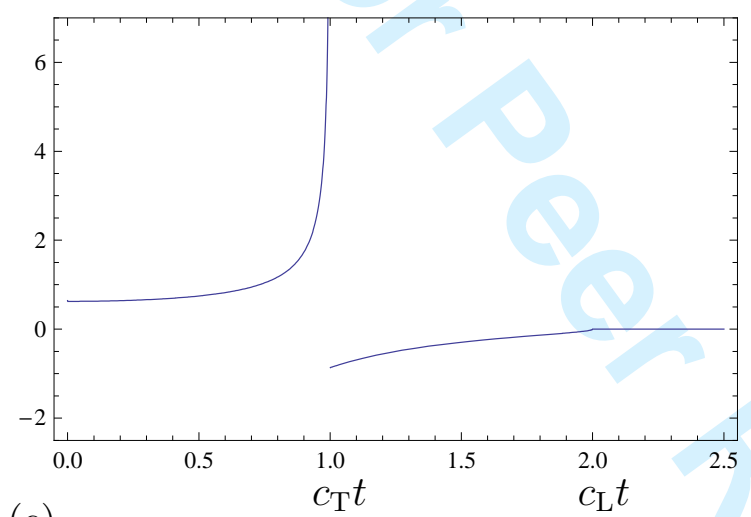

(c)

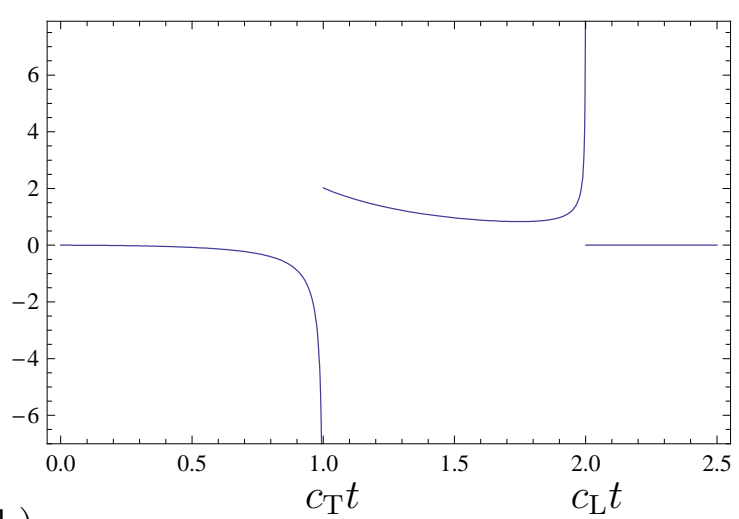

(b)

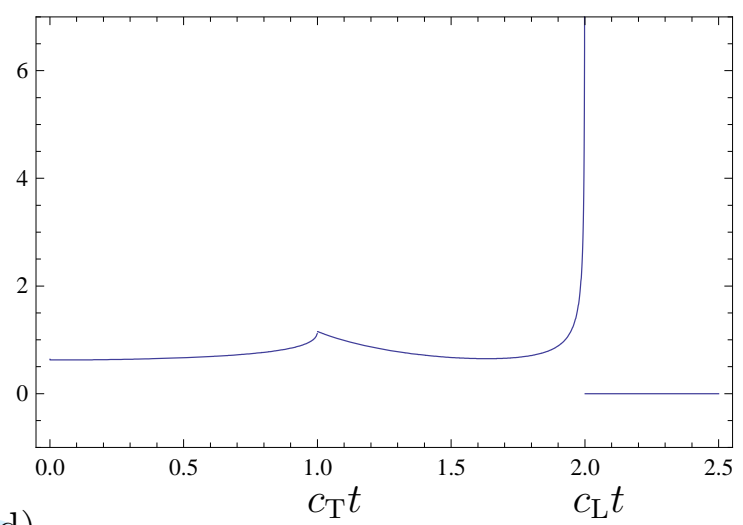

(d)

Figure 1: Two-dimensional Green tensors as functions of $r$ for fixed time $t$ : (a) $G_{z z}$, (b) $G_{x y}$ for $\varphi=\pi / 4$, (c) $G_{x x}$ for $\varphi=\pi / 2$ and (d) $G_{x x}$ for $\varphi=0$. In the plots we used $c_{\mathrm{T}}=1$ and $c_{\mathrm{L}}=2$ for numeric convenience.

\section{Screw dislocation}

Let us consider a nonuniformly moving screw dislocation at the position $(\xi(t), \eta(t))$ at time $t$. The dislocation line and the Burgers vector $b_{z}$ are parallel to the $z$-axis. The components of the dislocation velocity are: $V_{x}=\dot{\xi}(t), V_{y}=\dot{\eta}(t)$. The non-vanishing components of the dislocation density and dislocation current tensors are

$$
\begin{aligned}
T_{z x y} & =b_{z} \delta(x-\xi(t)) \delta(y-\eta(t)) \\
I_{z x} & =V_{y} T_{z x y} \\
I_{z y} & =-V_{x} T_{z x y}
\end{aligned}
$$


For a screw dislocation, Eqs. (17) and (18) reduce to the non-vanishing components of the elastic fields

$$
\begin{aligned}
v_{z}(\boldsymbol{r}, t)= & \partial_{x} \int_{-\infty}^{t} \int_{-\infty}^{\infty} C_{z x z x} G_{z z}\left(\boldsymbol{r}-\boldsymbol{r}^{\prime}, t-t^{\prime}\right) I_{z x}\left(\boldsymbol{r}^{\prime}, t^{\prime}\right) \mathrm{d} \boldsymbol{r}^{\prime} \mathrm{d} t^{\prime} \\
& +\partial_{y} \int_{-\infty}^{t} \int_{-\infty}^{\infty} C_{z y z y} G_{z z}\left(\boldsymbol{r}-\boldsymbol{r}^{\prime}, t-t^{\prime}\right) I_{z y}\left(\boldsymbol{r}^{\prime}, t^{\prime}\right) \mathrm{d} \boldsymbol{r}^{\prime} \mathrm{d} t^{\prime}, \\
\beta_{z x}(\boldsymbol{r}, t)= & \partial_{y} \int_{-\infty}^{t} \int_{-\infty}^{\infty} C_{z y z y} G_{z z}\left(\boldsymbol{r}-\boldsymbol{r}^{\prime}, t-t^{\prime}\right) T_{z x y}\left(\boldsymbol{r}^{\prime}, t^{\prime}\right) \mathrm{d} \boldsymbol{r}^{\prime} \mathrm{d} t^{\prime} \\
& +\partial_{t} \int_{-\infty}^{t} \int_{-\infty}^{\infty} \rho G_{z z}\left(\boldsymbol{r}-\boldsymbol{r}^{\prime}, t-t^{\prime}\right) I_{z x}\left(\boldsymbol{r}^{\prime}, t^{\prime}\right) \mathrm{d} \boldsymbol{r}^{\prime} \mathrm{d} t^{\prime} \\
\beta_{z y}(\boldsymbol{r}, t)= & \partial_{x} \int_{-\infty}^{t} \int_{-\infty}^{\infty} C_{z x z x} G_{z z}\left(\boldsymbol{r}-\boldsymbol{r}^{\prime}, t-t^{\prime}\right) T_{z y x}\left(\boldsymbol{r}^{\prime}, t^{\prime}\right) \mathrm{d} \boldsymbol{r}^{\prime} \mathrm{d} t^{\prime} \\
& +\partial_{t} \int_{-\infty}^{t} \int_{-\infty}^{\infty} \rho G_{z z}\left(\boldsymbol{r}-\boldsymbol{r}^{\prime}, t-t^{\prime}\right) I_{z y}\left(\boldsymbol{r}^{\prime}, t^{\prime}\right) \mathrm{d} \boldsymbol{r}^{\prime} \mathrm{d} t^{\prime}
\end{aligned}
$$

Substituting Eqs. (20), (23) and (25)-(27) into Eqs. (28)-(30) and carrying out the integration in $\boldsymbol{r}^{\prime}$, we find the following time integral representations

$$
\begin{aligned}
v_{z} & =A_{y, x}-A_{x, y}, \\
\beta_{z x} & =\phi_{, y}+\frac{1}{c_{\mathrm{T}}^{2}} \dot{A}_{y}, \\
\beta_{z y} & =-\left(\phi_{, x}+\frac{1}{c_{\mathrm{T}}^{2}} \dot{A}_{x}\right),
\end{aligned}
$$

where the 'potential' functions are defined by

$$
\begin{aligned}
A_{i} & =\mu b_{z} \int_{-\infty}^{t} V_{i}\left(t^{\prime}\right) G_{z z}\left(\boldsymbol{r}-\boldsymbol{r}^{\prime \prime}\left(t^{\prime}\right), t-t^{\prime}\right) \mathrm{d} t^{\prime}, \\
\phi & =\mu b_{z} \int_{-\infty}^{t} G_{z z}\left(\boldsymbol{r}-\boldsymbol{r}^{\prime \prime}\left(t^{\prime}\right), t-t^{\prime}\right) \mathrm{d} t^{\prime}
\end{aligned}
$$

with $\boldsymbol{r}^{\prime \prime}=(\xi, \eta)$. Substituting the Green function (23) in Eqs. (34) and (35), the 'potential' functions read

$$
\begin{aligned}
A_{x} & =\frac{b_{z}}{2 \pi} \int_{-\infty}^{t_{\mathrm{T}}} \frac{V_{x}\left(t^{\prime}\right)}{S_{\mathrm{T}}} \mathrm{d} t^{\prime}, \\
A_{y} & =\frac{b_{z}}{2 \pi} \int_{-\infty}^{t_{\mathrm{T}}} \frac{V_{y}\left(t^{\prime}\right)}{S_{\mathrm{T}}} \mathrm{d} t^{\prime}, \\
\phi & =\frac{b_{z}}{2 \pi} \int_{-\infty}^{t_{\mathrm{T}}} \frac{1}{S_{\mathrm{T}}} \mathrm{d} t^{\prime}
\end{aligned}
$$

with the notations

$$
\begin{array}{ll}
\bar{x}=x-\xi\left(t^{\prime}\right), & \bar{y}=y-\eta\left(t^{\prime}\right) \quad \bar{t}=t-t^{\prime}, \quad \bar{R}^{2}=\bar{x}^{2}+\bar{y}^{2}, \\
S_{\mathrm{T}}^{2}=\bar{t}^{2}-\frac{\bar{R}^{2}}{c_{\mathrm{T}}^{2}}, & t_{\mathrm{T}}=t-\frac{\bar{R}}{c_{\mathrm{T}}} .
\end{array}
$$


The 'potential' functions (36)-(38) play the physical role of the retarded potentials of a screw dislocation moving nonuniformly. Note that $t_{\mathrm{T}}$ is called retarded time, which is the root of the equation $S_{\mathrm{T}}^{2}=0$ and is less than $t$, and $(\bar{x}, \bar{y})$ is the distance between the field point $(x, y)$ and the position $(\xi, \eta)$ of the dislocation. Here, $\xi\left(t^{\prime}\right)$ and $\eta\left(t^{\prime}\right)$ are the positions in the $x$ and $y$ directions of the dislocation at time $t^{\prime}, V_{x}\left(t^{\prime}\right)$ and $V_{y}\left(t^{\prime}\right)$ are the velocity components of the dislocation at the same time $t^{\prime}$. The retarded time $t_{\mathrm{T}}$ is the time before $t$, when the dislocation caused an excitation of the elastic field, which moves from $(\xi, \eta)$ to $(x, y)$ in the time $\bar{R} / c_{\mathrm{T}}$. Thus, $t-t_{\mathrm{T}}=\bar{R} / c_{\mathrm{T}}$ is the time if the elastic dislocation fields move from $(\xi, \eta)$ to $(x, y)$ with velocity $c_{\mathrm{T}}$. Eqs. (31)-(38) agree with the expressions given by Eshelby [11] (see also [9, 17]). Eshelby [11] derived the elastic fields using an electromagnetic analogy. Here we derived the elastic fields in a more straightforward manner as solutions of the equations of motion (15) and (16). The solutions $(31)-(38)$ on the position $(x, y)$ at time $t$ depend on all contributions emitted at all times $t^{\prime}$ from $-\infty$ up to $t_{\mathrm{T}}$. That is why Eshelby [10] said: 'The dislocation is haunted by its past'. The physical interpretation is that disturbances from remote positions on the dislocation line continue to arrive at other positions on the line as the dislocation moves forward. Thus, the motion of a screw dislocation produces only transversal elastic waves.

\section{Gliding edge dislocation}

In this section, we investigate the problem of an edge dislocation at the position $(\xi(t), 0)$ at time $t$ gliding in $x$-direction with arbitrary dislocation velocity $V_{x}(t)=\dot{\xi}(t)$ and Burgers vector $b_{x}$. The non-vanishing components of the dislocation density and dislocation current tensors read

$$
\begin{aligned}
T_{x x y} & =b_{x} \delta(x-\xi(t)) \delta(y), \\
I_{x y} & =-V_{x} T_{x x y} .
\end{aligned}
$$

For a gliding edge dislocation, Eqs. (17) and (18) reduce to the non-vanishing components

$$
\begin{aligned}
v_{x}(\boldsymbol{r}, t)= & \partial_{y} \int_{-\infty}^{t} \int_{-\infty}^{\infty} C_{x y x y} G_{x x}\left(\boldsymbol{r}-\boldsymbol{r}^{\prime}, t-t^{\prime}\right) I_{x y}\left(\boldsymbol{r}^{\prime}, t^{\prime}\right) \mathrm{d} \boldsymbol{r}^{\prime} \mathrm{d} t^{\prime} \\
& +\partial_{x} \int_{-\infty}^{t} \int_{-\infty}^{\infty} C_{y x x y} G_{x y}\left(\boldsymbol{r}-\boldsymbol{r}^{\prime}, t-t^{\prime}\right) I_{x y}\left(\boldsymbol{r}^{\prime}, t^{\prime}\right) \mathrm{d} \boldsymbol{r}^{\prime} \mathrm{d} t^{\prime}, \\
v_{y}(\boldsymbol{r}, t)= & \partial_{x} \int_{-\infty}^{t} \int_{-\infty}^{\infty} C_{y x x y} G_{y y}\left(\boldsymbol{r}-\boldsymbol{r}^{\prime}, t-t^{\prime}\right) I_{x y}\left(\boldsymbol{r}^{\prime}, t^{\prime}\right) \mathrm{d} \boldsymbol{r}^{\prime} \mathrm{d} t^{\prime} \\
& +\partial_{y} \int_{-\infty}^{t} \int_{-\infty}^{\infty} C_{x y x y} G_{y x}\left(\boldsymbol{r}-\boldsymbol{r}^{\prime}, t-t^{\prime}\right) I_{x y}\left(\boldsymbol{r}^{\prime}, t^{\prime}\right) \mathrm{d} \boldsymbol{r}^{\prime} \mathrm{d} t^{\prime}
\end{aligned}
$$


and

$$
\begin{aligned}
\beta_{x x}(\boldsymbol{r}, t)= & \partial_{y} \int_{-\infty}^{t} \int_{-\infty}^{\infty} C_{x y x y} G_{x x}\left(\boldsymbol{r}-\boldsymbol{r}^{\prime}, t-t^{\prime}\right) T_{x x y}\left(\boldsymbol{r}^{\prime}, t^{\prime}\right) \mathrm{d} \boldsymbol{r}^{\prime} \mathrm{d} t^{\prime} \\
& +\partial_{x} \int_{-\infty}^{t} \int_{-\infty}^{\infty} C_{y x x y} G_{x y}\left(\boldsymbol{r}-\boldsymbol{r}^{\prime}, t-t^{\prime}\right) T_{x x y}\left(\boldsymbol{r}^{\prime}, t^{\prime}\right) \mathrm{d} \boldsymbol{r}^{\prime} \mathrm{d} t^{\prime} \\
\beta_{y x}(\boldsymbol{r}, t)= & \partial_{y} \int_{-\infty}^{t} \int_{-\infty}^{\infty} C_{x y x y} G_{y x}\left(\boldsymbol{r}-\boldsymbol{r}^{\prime}, t-t^{\prime}\right) T_{x x y}\left(\boldsymbol{r}^{\prime}, t^{\prime}\right) \mathrm{d} \boldsymbol{r}^{\prime} \mathrm{d} t^{\prime} \\
& +\partial_{x} \int_{-\infty}^{t} \int_{-\infty}^{\infty} C_{y x x y} G_{y y}\left(\boldsymbol{r}-\boldsymbol{r}^{\prime}, t-t^{\prime}\right) T_{x x y}\left(\boldsymbol{r}^{\prime}, t^{\prime}\right) \mathrm{d} \boldsymbol{r}^{\prime} \mathrm{d} t^{\prime} \\
\beta_{x y}(\boldsymbol{r}, t)= & \partial_{x} \int_{-\infty}^{t} \int_{-\infty}^{\infty} C_{x x x x} G_{x x}\left(\boldsymbol{r}-\boldsymbol{r}^{\prime}, t-t^{\prime}\right) T_{x y x}\left(\boldsymbol{r}^{\prime}, t^{\prime}\right) \mathrm{d} \boldsymbol{r}^{\prime} \mathrm{d} t^{\prime} \\
& +\partial_{y} \int_{-\infty}^{t} \int_{-\infty}^{\infty} C_{y y x x} G_{x y}\left(\boldsymbol{r}-\boldsymbol{r}^{\prime}, t-t^{\prime}\right) T_{x y x}\left(\boldsymbol{r}^{\prime}, t^{\prime}\right) \mathrm{d} \boldsymbol{r}^{\prime} \mathrm{d} t^{\prime} \\
& +\partial_{t} \int_{-\infty}^{t} \int_{-\infty}^{\infty} \rho G_{x x}\left(\boldsymbol{r}-\boldsymbol{r}^{\prime}, t-t^{\prime}\right) I_{x y}\left(\boldsymbol{r}^{\prime}, t^{\prime}\right) \mathrm{d} \boldsymbol{r}^{\prime} \mathrm{d} t^{\prime}, \\
\beta_{y y}(\boldsymbol{r}, t)= & \partial_{x} \int_{-\infty}^{t} \int_{-\infty}^{\infty} C_{x x x x} G_{y x}\left(\boldsymbol{r}-\boldsymbol{r}^{\prime}, t-t^{\prime}\right) T_{x y x}\left(\boldsymbol{r}^{\prime}, t^{\prime}\right) \mathrm{d} \boldsymbol{r}^{\prime} \mathrm{d} t^{\prime} \\
& +\partial_{y} \int_{-\infty}^{t} \int_{-\infty}^{\infty} C_{y y x x} G_{y y}\left(\boldsymbol{r}-\boldsymbol{r}^{\prime}, t-t^{\prime}\right) T_{x y x}\left(\boldsymbol{r}^{\prime}, t^{\prime}\right) \mathrm{d} \boldsymbol{r}^{\prime} \mathrm{d} t^{\prime} \\
& +\partial_{t} \int_{-\infty}^{t} \int_{-\infty}^{\infty} \rho G_{y x}\left(\boldsymbol{r}-\boldsymbol{r}^{\prime}, t-t^{\prime}\right) I_{x y}\left(\boldsymbol{r}^{\prime}, t^{\prime}\right) \mathrm{d} \boldsymbol{r}^{\prime} \mathrm{d} t^{\prime} .
\end{aligned}
$$

Substituting Eqs. (20), (22), (40) and (41) in Eqs. (42)-(47) and carrying out the integration in $\boldsymbol{r}^{\prime}$, we find the following time integral representations for the elastic fields of a gliding edge dislocation

$$
\begin{aligned}
& v_{x}=-\frac{b_{x} c_{\mathrm{T}}^{2}}{2 \pi}\left(A_{x y, x}+A_{x x, y}\right), \\
& v_{y}=-\frac{b_{x} c_{\mathrm{T}}^{2}}{2 \pi}\left(A_{y y, x}+A_{y x, y}\right),
\end{aligned}
$$

and

$$
\begin{aligned}
& \beta_{x x}=\frac{b_{x} c_{\mathrm{T}}^{2}}{2 \pi}\left(\phi_{x y, x}+\phi_{x x, y}\right), \\
& \beta_{y x}=\frac{b_{x} c_{\mathrm{T}}^{2}}{2 \pi}\left(\phi_{y y, x}+\phi_{y x, y}\right), \\
& \beta_{x y}=-\frac{b_{x} c_{\mathrm{L}}^{2}}{2 \pi}\left(\phi_{x x, x}+\phi_{x y, y}\right)+\frac{b_{x} c_{\mathrm{T}}^{2}}{\pi} \phi_{x y, y}-\frac{b_{x}}{2 \pi} \dot{A}_{x x}, \\
& \beta_{y y}=-\frac{b_{x} c_{\mathrm{L}}^{2}}{2 \pi}\left(\phi_{y x, x}+\phi_{y y, y}\right)+\frac{b_{x} c_{\mathrm{T}}^{2}}{\pi} \phi_{y y, y}-\frac{b_{x}}{2 \pi} \dot{A}_{y x},
\end{aligned}
$$


where the following 'potential' functions are defined by

$$
\begin{aligned}
& A_{i j}=2 \pi \rho \int_{-\infty}^{t} V_{x}\left(t^{\prime}\right) G_{i j}\left(\boldsymbol{r}-\boldsymbol{r}^{\prime \prime}\left(t^{\prime}\right), t-t^{\prime}\right) \mathrm{d} t^{\prime}, \\
& \phi_{i j}=2 \pi \rho \int_{-\infty}^{t} G_{i j}\left(\boldsymbol{r}-\boldsymbol{r}^{\prime \prime}\left(t^{\prime}\right), t-t^{\prime}\right) \mathrm{d} t^{\prime}
\end{aligned}
$$

with $\boldsymbol{r}^{\prime \prime}=(\xi, 0), A_{i j}=A_{j i}$ and $\phi_{i j}=\phi_{j i}$. Substituting the Green tensor (22), they finally read

$$
\begin{aligned}
& A_{x x}=\int_{-\infty}^{t_{\mathrm{L}}} \frac{V_{x}\left(t^{\prime}\right)}{\bar{R}^{4}}\left(\frac{\bar{x}^{2} \bar{t}^{2}}{S_{\mathrm{L}}}-\bar{y}^{2} S_{\mathrm{L}}\right) \mathrm{d} t^{\prime}+\int_{-\infty}^{t_{\mathrm{T}}} \frac{V_{x}\left(t^{\prime}\right)}{\bar{R}^{4}}\left(\frac{\bar{y}^{2} \bar{t}^{2}}{S_{\mathrm{T}}}-\bar{x}^{2} S_{\mathrm{T}}\right) \mathrm{d} t^{\prime}, \\
& A_{y y}=\int_{-\infty}^{t_{\mathrm{L}}} \frac{V_{x}\left(t^{\prime}\right)}{\bar{R}^{4}}\left(\frac{\bar{y}^{2} \bar{t}^{2}}{S_{\mathrm{L}}}-\bar{x}^{2} S_{\mathrm{L}}\right) \mathrm{d} t^{\prime}+\int_{-\infty}^{t_{\mathrm{T}}} \frac{V_{x}\left(t^{\prime}\right)}{\bar{R}^{4}}\left(\frac{\bar{x}^{2} \bar{t}^{2}}{S_{\mathrm{T}}}-\bar{y}^{2} S_{\mathrm{T}}\right) \mathrm{d} t^{\prime}, \\
& A_{x y}=\int_{-\infty}^{t_{\mathrm{L}}} \frac{V_{x}\left(t^{\prime}\right)}{\bar{R}^{4}} \bar{x} \bar{y}\left(\frac{\bar{t}^{2}}{S_{\mathrm{L}}}+S_{\mathrm{L}}\right) \mathrm{d} t^{\prime}-\int_{-\infty}^{t_{\mathrm{T}}} \frac{V_{x}\left(t^{\prime}\right)}{\bar{R}^{4}} \bar{x} \bar{y}\left(\frac{\bar{t}^{2}}{S_{\mathrm{T}}}+S_{\mathrm{T}}\right) \mathrm{d} t^{\prime},
\end{aligned}
$$

with

$$
\begin{aligned}
& \bar{x}=x-\xi\left(t^{\prime}\right), \quad \bar{y}=y \quad \bar{t}=t-t^{\prime}, \quad \bar{R}^{2}=\bar{x}^{2}+\bar{y}^{2}, \\
& S_{\mathrm{T}}^{2}=\bar{t}^{2}-\frac{\bar{R}^{2}}{c_{\mathrm{T}}^{2}}, \quad t_{\mathrm{T}}=t-\frac{\bar{R}}{c_{\mathrm{T}}}, \quad S_{\mathrm{L}}^{2}=\bar{t}^{2}-\frac{\bar{R}^{2}}{c_{\mathrm{L}}^{2}}, \quad t_{\mathrm{L}}=t-\frac{\bar{R}}{c_{\mathrm{L}}} .
\end{aligned}
$$

The explicit expressions for $\phi_{i j}$ can be obtained from the corresponding expressions for $A_{i j}$ if $V_{x}\left(t^{\prime}\right)$ is replaced by 1 . If we do this replacement, we observe the relations: $v_{x}\left(V_{x}\left(t^{\prime}\right)=\right.$ $1)=-\beta_{x x}$ and $v_{y}\left(V_{x}\left(t^{\prime}\right)=1\right)=-\beta_{y x}$. The potential functions $A_{i j}$ and $\phi_{i j}$ are the retarded potentials for a gliding edge dislocation. The elastic fields of a gliding edge dislocation are given as time integrals and they are more complicated than the expressions for a screw dislocation. The elastic fields contain terms proportional to $S_{\mathrm{T}}, S_{\mathrm{L}}, 1 / S_{\mathrm{T}}$ and $1 / S_{\mathrm{L}}$. The appearance of two velocities of elastic waves $c_{\mathrm{T}}$ and $c_{\mathrm{L}}$ makes the results more complicated and is also the reason of two retarded times $t_{\mathrm{T}}$ and $t_{\mathrm{L}}$ which are the roots of $S_{\mathrm{T}}^{2}=0$ and $S_{\mathrm{L}}^{2}=0$, respectively. Eqs. (48)-(58) agree with the expressions given by Kiusalaas and Mura [14]. For the calculation of the elastic fields, Kiusalaas and Mura [14] used the threedimensional Green tensor in contrast to the two-dimensional Green tensor (22) that is used in the present paper thereby making the calculation easier and more straightforward as well as appropriate for the calculation of the elastic fields of a climbing edge dislocation. The structure of the potential functions (56)-(58) is immediately a consequence of the form of the two-dimensional Green tensor (22). It is clear that the gliding of the edge dislocation causes transversal as well as longitudinal elastic waves with the velocities $c_{\mathrm{T}}$ and $c_{\mathrm{L}}$, respectively.

\section{Climbing edge dislocation}

In this section, for the first time we investigate the problem of a climbing edge dislocation at the position $(0, \eta(t))$ at time $t$ climbing in $y$-direction with arbitrary dislocation velocity 
$V_{y}(t)=\dot{\eta}(t)$ and Burgers vector $b_{x}$. The non-vanishing components of the dislocation density and dislocation current tensors are given by

$$
\begin{aligned}
T_{x x y} & =b_{x} \delta(x) \delta(y-\eta(t)), \\
I_{x x} & =V_{y} T_{x x y} .
\end{aligned}
$$

Thus, for a climbing edge dislocation, Eqs. (17) and (18) reduce to the non-vanishing components of the elastic fields

$$
\begin{aligned}
v_{x}(\boldsymbol{r}, t)= & \partial_{x} \int_{-\infty}^{t} \int_{-\infty}^{\infty} C_{x x x x} G_{x x}\left(\boldsymbol{r}-\boldsymbol{r}^{\prime}, t-t^{\prime}\right) I_{x x}\left(\boldsymbol{r}^{\prime}, t^{\prime}\right) \mathrm{d} \boldsymbol{r}^{\prime} \mathrm{d} t^{\prime} \\
& +\partial_{y} \int_{-\infty}^{t} \int_{-\infty}^{\infty} C_{y y x x} G_{x y}\left(\boldsymbol{r}-\boldsymbol{r}^{\prime}, t-t^{\prime}\right) I_{x x}\left(\boldsymbol{r}^{\prime}, t^{\prime}\right) \mathrm{d} \boldsymbol{r}^{\prime} \mathrm{d} t^{\prime}, \\
v_{y}(\boldsymbol{r}, t)= & \partial_{x} \int_{-\infty}^{t} \int_{-\infty}^{\infty} C_{x x x x} G_{y x}\left(\boldsymbol{r}-\boldsymbol{r}^{\prime}, t-t^{\prime}\right) I_{x x}\left(\boldsymbol{r}^{\prime}, t^{\prime}\right) \mathrm{d} \boldsymbol{r}^{\prime} \mathrm{d} t^{\prime} \\
& +\partial_{y} \int_{-\infty}^{t} \int_{-\infty}^{\infty} C_{y y x x} G_{y y}\left(\boldsymbol{r}-\boldsymbol{r}^{\prime}, t-t^{\prime}\right) I_{x x}\left(\boldsymbol{r}^{\prime}, t^{\prime}\right) \mathrm{d} \boldsymbol{r}^{\prime} \mathrm{d} t^{\prime}
\end{aligned}
$$

and

$$
\begin{aligned}
\beta_{x x}(\boldsymbol{r}, t)= & \partial_{y} \int_{-\infty}^{t} \int_{-\infty}^{\infty} C_{x y x y} G_{x x}\left(\boldsymbol{r}-\boldsymbol{r}^{\prime}, t-t^{\prime}\right) T_{x x y}\left(\boldsymbol{r}^{\prime}, t^{\prime}\right) \mathrm{d} \boldsymbol{r}^{\prime} \mathrm{d} t^{\prime} \\
& +\partial_{x} \int_{-\infty}^{t} \int_{-\infty}^{\infty} C_{y x x y} G_{x y}\left(\boldsymbol{r}-\boldsymbol{r}^{\prime}, t-t^{\prime}\right) T_{x x y}\left(\boldsymbol{r}^{\prime}, t^{\prime}\right) \mathrm{d} \boldsymbol{r}^{\prime} \mathrm{d} t^{\prime} \\
& +\partial_{t} \int_{-\infty}^{t} \int_{-\infty}^{\infty} \rho G_{x x}\left(\boldsymbol{r}-\boldsymbol{r}^{\prime}, t-t^{\prime}\right) I_{x x}\left(\boldsymbol{r}^{\prime}, t^{\prime}\right) \mathrm{d} \boldsymbol{r}^{\prime} \mathrm{d} t^{\prime}, \\
\beta_{y x}(\boldsymbol{r}, t)= & \partial_{y} \int_{-\infty}^{t} \int_{-\infty}^{\infty} C_{x y x y} G_{y x}\left(\boldsymbol{r}-\boldsymbol{r}^{\prime}, t-t^{\prime}\right) T_{x x y}\left(\boldsymbol{r}^{\prime}, t^{\prime}\right) \mathrm{d} \boldsymbol{r}^{\prime} \mathrm{d} t^{\prime} \\
& +\partial_{x} \int_{-\infty}^{t} \int_{-\infty}^{\infty} C_{y x x y} G_{y y}\left(\boldsymbol{r}-\boldsymbol{r}^{\prime}, t-t^{\prime}\right) T_{x x y}\left(\boldsymbol{r}^{\prime}, t^{\prime}\right) \mathrm{d} \boldsymbol{r}^{\prime} \mathrm{d} t^{\prime} \\
& +\partial_{t} \int_{-\infty}^{t} \int_{-\infty}^{\infty} \rho G_{y x}\left(\boldsymbol{r}-\boldsymbol{r}^{\prime}, t-t^{\prime}\right) I_{x x}\left(\boldsymbol{r}^{\prime}, t^{\prime}\right) \mathrm{d} \boldsymbol{r}^{\prime} \mathrm{d} t^{\prime}, \\
\beta_{x y}(\boldsymbol{r}, t)= & \partial_{x} \int_{-\infty}^{t} \int_{-\infty}^{\infty} C_{x x x x} G_{x x}\left(\boldsymbol{r}-\boldsymbol{r}^{\prime}, t-t^{\prime}\right) T_{x y x}\left(\boldsymbol{r}^{\prime}, t^{\prime}\right) \mathrm{d} \boldsymbol{r}^{\prime} \mathrm{d} t^{\prime} \\
& +\partial_{y} \int_{-\infty}^{t} \int_{-\infty}^{\infty} C_{y y x x} G_{x y}\left(\boldsymbol{r}-\boldsymbol{r}^{\prime}, t-t^{\prime}\right) T_{x y x}\left(\boldsymbol{r}^{\prime}, t^{\prime}\right) \mathrm{d} \boldsymbol{r}^{\prime} \mathrm{d} t^{\prime}, \\
& +\partial_{x} \int_{-\infty}^{t} \int_{-\infty}^{\infty} C_{x x x x} G_{y x}\left(\boldsymbol{r}-\boldsymbol{r}^{\prime}, t-t^{\prime}\right) T_{x y x}\left(\boldsymbol{r}^{\prime}, t^{\prime}\right) \mathrm{d} \boldsymbol{r}^{\prime} \mathrm{d} t^{\prime} \\
\beta_{y y}(\boldsymbol{r}, t) & \int_{-\infty}^{\infty} C_{y y x x} G_{y y}\left(\boldsymbol{r}-\boldsymbol{r}^{\prime}, t-t^{\prime}\right) T_{x y x}\left(\boldsymbol{r}^{\prime}, t^{\prime}\right) \mathrm{d} \boldsymbol{r}^{\prime} \mathrm{d} t^{\prime} .
\end{aligned}
$$

If we substitute Eqs. (20), (22), (60) and (61) in Eqs. (62)-(67) and we carry out the integration in $\boldsymbol{r}^{\prime}$, we obtain the following time integral representations for the elastic fields 
of a climbing edge dislocation

$$
\begin{aligned}
& v_{x}=\frac{b_{x} c_{\mathrm{L}}^{2}}{2 \pi}\left(A_{x x, x}+A_{x y, y}\right)-\frac{b_{x} c_{\mathrm{T}}^{2}}{\pi} A_{x y, y}, \\
& v_{y}=\frac{b_{x} c_{\mathrm{L}}^{2}}{2 \pi}\left(A_{y x, x}+A_{y y, y}\right)-\frac{b_{x} c_{\mathrm{T}}^{2}}{\pi} A_{y y, y},
\end{aligned}
$$

and

$$
\begin{aligned}
& \beta_{x x}=\frac{b_{x} c_{\mathrm{T}}^{2}}{2 \pi}\left(\phi_{x y, x}+\phi_{x x, y}\right)+\frac{b_{x}}{2 \pi} \dot{A}_{x x}, \\
& \beta_{y x}=\frac{b_{x} c_{\mathrm{T}}^{2}}{2 \pi}\left(\phi_{y y, x}+\phi_{y x, y}\right)+\frac{b_{x}}{2 \pi} \dot{A}_{y x} \\
& \beta_{x y}=-\frac{b_{x} c_{\mathrm{L}}^{2}}{2 \pi}\left(\phi_{x x, x}+\phi_{x y, y}\right)+\frac{b_{x} c_{\mathrm{T}}^{2}}{\pi} \phi_{x y, y}, \\
& \beta_{y y}=-\frac{b_{x} c_{\mathrm{L}}^{2}}{2 \pi}\left(\phi_{y x, x}+\phi_{y y, y}\right)+\frac{b_{x} c_{\mathrm{T}}^{2}}{\pi} \phi_{y y, y},
\end{aligned}
$$

where the 'potential' functions are defined by

$$
\begin{aligned}
& A_{i j}=2 \pi \rho \int_{-\infty}^{t} V_{y}\left(t^{\prime}\right) G_{i j}\left(\boldsymbol{r}-\boldsymbol{r}^{\prime \prime}\left(t^{\prime}\right), t-t^{\prime}\right) \mathrm{d} t^{\prime}, \\
& \phi_{i j}=2 \pi \rho \int_{-\infty}^{t} G_{i j}\left(\boldsymbol{r}-\boldsymbol{r}^{\prime \prime}\left(t^{\prime}\right), t-t^{\prime}\right) \mathrm{d} t^{\prime}
\end{aligned}
$$

where $\boldsymbol{r}^{\prime \prime}=(0, \eta), A_{i j}=A_{j i}$ and $\phi_{i j}=\phi_{j i}$. After substituting the Green tensor (22), they read

$$
\begin{aligned}
& A_{x x}=\int_{-\infty}^{t_{\mathrm{L}}} \frac{V_{y}\left(t^{\prime}\right)}{\bar{R}^{4}}\left(\frac{\bar{x}^{2} \bar{t}^{2}}{S_{\mathrm{L}}}-\bar{y}^{2} S_{\mathrm{L}}\right) \mathrm{d} t^{\prime}+\int_{-\infty}^{t_{\mathrm{T}}} \frac{V_{y}\left(t^{\prime}\right)}{\bar{R}^{4}}\left(\frac{\bar{y}^{2} \bar{t}^{2}}{S_{\mathrm{T}}}-\bar{x}^{2} S_{\mathrm{T}}\right) \mathrm{d} t^{\prime}, \\
& A_{y y}=\int_{-\infty}^{t_{\mathrm{L}}} \frac{V_{y}\left(t^{\prime}\right)}{\bar{R}^{4}}\left(\frac{\bar{y}^{2} \bar{t}^{2}}{S_{\mathrm{L}}}-\bar{x}^{2} S_{\mathrm{L}}\right) \mathrm{d} t^{\prime}+\int_{-\infty}^{t_{\mathrm{T}}} \frac{V_{y}\left(t^{\prime}\right)}{\bar{R}^{4}}\left(\frac{\bar{x}^{2} \bar{t}^{2}}{S_{\mathrm{T}}}-\bar{y}^{2} S_{\mathrm{T}}\right) \mathrm{d} t^{\prime}, \\
& A_{x y}=\int_{-\infty}^{t_{\mathrm{L}}} \frac{V_{y}\left(t^{\prime}\right)}{\bar{R}^{4}} \bar{x} \bar{y}\left(\frac{\bar{t}^{2}}{S_{\mathrm{L}}}+S_{\mathrm{L}}\right) \mathrm{d} t^{\prime}-\int_{-\infty}^{t_{\mathrm{T}}} \frac{V_{y}\left(t^{\prime}\right)}{\bar{R}^{4}} \bar{x} \bar{y}\left(\frac{\bar{t}^{2}}{S_{\mathrm{T}}}+S_{\mathrm{T}}\right) \mathrm{d} t^{\prime},
\end{aligned}
$$

with

$$
\begin{aligned}
& \bar{x}=x, \quad \bar{y}=y-\eta\left(t^{\prime}\right) \quad \bar{t}=t-t^{\prime}, \quad \bar{R}^{2}=\bar{x}^{2}+\bar{y}^{2}, \\
& S_{\mathrm{T}}^{2}=\bar{t}^{2}-\frac{\bar{R}^{2}}{c_{\mathrm{T}}^{2}}, \quad t_{\mathrm{T}}=t-\frac{\bar{R}}{c_{\mathrm{T}}}, \quad S_{\mathrm{L}}^{2}=\bar{t}^{2}-\frac{\bar{R}^{2}}{c_{\mathrm{L}}^{2}}, \quad t_{\mathrm{L}}=t-\frac{\bar{R}}{c_{\mathrm{L}}} .
\end{aligned}
$$

The explicit expressions for $\phi_{i j}$ can be obtained from the corresponding expressions for $A_{i j}$ replacing $V_{y}\left(t^{\prime}\right)$ by 1 . Using this replacement, we observe the relations: $v_{x}\left(V_{y}\left(t^{\prime}\right)=\right.$ $1)=-\beta_{x y}$ and $v_{y}\left(V_{y}\left(t^{\prime}\right)=1\right)=-\beta_{y y}$. Let us now discuss qualitatively the results. The expressions for a climbing edge dislocation (68)-(78) are similar to, but also different in some sense than, the expressions of a gliding edge dislocation (48)-(58). In particular, the elastic fields contain terms again proportional to $S_{\mathrm{T}}, S_{\mathrm{L}}, 1 / S_{\mathrm{T}}$ and $1 / S_{\mathrm{L}}$. In the potential 
functions (76)-(78) the dislocation velocity is $V_{y}\left(t^{\prime}\right)$ similar to $V_{x}\left(t^{\prime}\right)$ in Eqs. (56)-(58). The complexity and the type of integrals for a climbing edge dislocation in Eqs. (76)-(78) are the same as for a gliding edge dislocation in Eqs. (56)-(58). The structure of entering of the potential functions $\phi_{i j}$ in Eqs. (70)-(73) is the same as in Eqs. (50)-(53). However, there are some differences concerning the structure of entering of the potential functions $A_{i j}$ in Eqs. (68)-(73) compared with Eqs. (48)-(53). Of course, the differences in the elastic fields (50)-(53) and (70)-(73) are expected due to the kind (gliding - climbing) of the edge dislocation. The difference between the elastic fields of a gliding edge dislocation and a climbing edge dislocation is mainly based on the specific component of the dislocation current tensor for gliding and climbing. For the gliding edge dislocation the component $I_{x y}$ (see Eq. (41)) enters the Eqs. (42), (43), (46) and (47). However, for the climbing edge dislocation the component $I_{x x}$ (see Eq. (61)) enters the Eqs. (62)-(65). What is also interesting and should be noted is the difference in the expressions for the velocity fields. In Eqs. (48) and (49) for the gliding edge dislocation only the transversal velocity $c_{\mathrm{T}}$ appears in the prefactors while in the prefactors of Eqs. (68) and (69) for the climbing edge dislocation both velocities $c_{\mathrm{T}}$ and $c_{\mathrm{L}}$ appear. Finally, the elastic fields of a climbing edge dislocation represent transversal and longitudinal elastic waves with the velocities $c_{\mathrm{T}}$ and $c_{\mathrm{L}}$, respectively.

\section{$6 \quad$ Static limit}

In this section, we give the static limit of the elastic distortions of nonuniformly moving dislocations as a check of the above results and to show the connection between the dynamic solutions in this paper and the static solutions given by deWit [32]. Because all the potential functions (34), (35), (54), (55), (74) and (75) are defined as integrals of the Green tensors of plane strain and anti-plane strain, we will carry out the static limit for the Green tensors. The static limit of the Green tensors is defined by (see, e.g., [6])

$$
G_{i j}\left(\boldsymbol{r}-\boldsymbol{r}^{\prime}\right)=\lim _{t \rightarrow \infty} \int_{-\infty}^{t} G_{i j}\left(\boldsymbol{r}-\boldsymbol{r}^{\prime}, t-t^{\prime}\right) \mathrm{d} t^{\prime}
$$

Although the obtained results provide the solutions of the elastic fields to the general motion of straight dislocations, their application to explicit problems demands some care to avoid the occurrence of divergent integrals. For the static limit it is possible to do this by supposing a limit $-T$ instead of $-\infty$. Then although $G_{z z}$ in Eq. (23) and $G_{i j}$ in Eq. (22) diverge if we let $T \rightarrow \infty$, their derivatives do not, so that the elastic distortions are welldefined in the static limit. The static limit of the derivative of the Green tensor (22) is calculated with

$$
G_{i j, k}\left(\boldsymbol{r}-\boldsymbol{r}^{\prime}\right)=\lim _{T \rightarrow \infty} \partial_{k} \int_{-T}^{t} G_{i j}\left(\boldsymbol{r}-\boldsymbol{r}^{\prime}, t-t^{\prime}\right) \mathrm{d} t^{\prime}
$$

as

$$
G_{i j, k}\left(\boldsymbol{r}-\boldsymbol{r}^{\prime}\right)=-\frac{1}{8 \pi \mu(1-\nu)}\left[(3-4 \nu) \delta_{i j} \frac{R_{k}}{R^{2}}-\delta_{i k} \frac{R_{j}}{R^{2}}-\delta_{j k} \frac{R_{i}}{R^{2}}+2 \frac{R_{i} R_{j} R_{k}}{R^{4}}\right],
$$


where $\boldsymbol{R}=\boldsymbol{r}-\boldsymbol{r}^{\prime}, \nu$ is Poisson's ratio and $\lambda=2 \mu \nu /(1-2 \nu)$. For the derivative of the Green function $G_{z z}$

$$
G_{z z, k}\left(\boldsymbol{r}-\boldsymbol{r}^{\prime}\right)=\lim _{T \rightarrow \infty} \partial_{k} \int_{-T}^{t} G_{z z}\left(\boldsymbol{r}-\boldsymbol{r}^{\prime}, t-t^{\prime}\right) \mathrm{d} t^{\prime}
$$

we obtain

$$
G_{z z, k}\left(\boldsymbol{r}-\boldsymbol{r}^{\prime}\right)=-\frac{1}{2 \pi \mu} \frac{R_{k}}{R^{2}}
$$

From Eq. (17) we recover the static Mura formula [4]

$$
\beta_{i m}(\boldsymbol{r})=\int_{-\infty}^{\infty} C_{j k l n} G_{i j, k}\left(\boldsymbol{r}-\boldsymbol{r}^{\prime}\right) T_{l m n}\left(\boldsymbol{r}^{\prime}\right) \mathrm{d} \boldsymbol{r}^{\prime}
$$

Substituting Eqs. (82) and (84) and the dislocation densities $T_{z x y}=b_{z} \delta(x) \delta(y)$ and $T_{x x y}=b_{x} \delta(x) \delta(y)$ into Eq. (85), we recover the expressions of the elastic distortion given by deWit [32]. The non-vanishing components of the elastic distortion of a screw dislocation are given by

$$
\begin{aligned}
& \beta_{z x}=-\frac{b_{z}}{2 \pi} \frac{y}{r^{2}}, \\
& \beta_{z y}=\frac{b_{z}}{2 \pi} \frac{x}{r^{2}},
\end{aligned}
$$

which are the static limits of Eqs. (32) and (33). The non-vanishing components of the elastic distortion tensor of an edge dislocation are obtained as

$$
\begin{aligned}
& \beta_{x x}=-\frac{b_{x}}{4 \pi(1-\nu)} \frac{y}{r^{2}}\left[(1-2 \nu)+2 \frac{x^{2}}{r^{2}}\right], \\
& \beta_{y x}=-\frac{b_{x}}{4 \pi(1-\nu)} \frac{x}{r^{2}}\left[(1-2 \nu)+2 \frac{y^{2}}{r^{2}}\right], \\
& \beta_{x y}=\frac{b_{x}}{4 \pi(1-\nu)} \frac{x}{r^{2}}\left[(3-2 \nu)-2 \frac{y^{2}}{r^{2}}\right], \\
& \beta_{y y}=-\frac{b_{x}}{4 \pi(1-\nu)} \frac{y}{r^{2}}\left[(1-2 \nu)-2 \frac{x^{2}}{r^{2}}\right],
\end{aligned}
$$

which are the static limits of the gliding edge dislocation (50)-(53) and the climbing edge dislocation (70)-(73).

\section{Acknowledgement}

The author gratefully acknowledges the grants of the Deutsche Forschungsgemeinschaft (Grant Nos. La1974/1-2, La1974/1-3, La1974/2-1). The author wishes to express his gratitude to Dr. Eleni Agiasofitou for her constructive and very helpful remarks during the preparation of the paper. The author also wants to thank two anonymous reviewers for their helpful remarks on an earlier version of the paper. 


\section{References}

[1] J. Weertman and J.R. Weertman, Moving dislocations. In: Dislocations in Solids, Vol. 3. F.R.N. Nabarro, ed., North-Holland, Amsterdam (1980) pp. 1-59.

[2] J.C. Savage, Dislocations in seismology. In: Dislocations in Solids, Vol. 3. F.R.N. Nabarro, ed., North-Holland, Amsterdam (1980) pp. 251-339.

[3] K. Aki and P.G. Richards, Quantitative Seismology. 2nd Edition, University Science Books (2002).

[4] T. Mura, Continuous distribution of moving dislocations. Phil. Mag. 8 (1963) 843-857.

[5] T. Mura, On dynamic problems of continuous distribution of dislocations. Int. J. Engng. Sci. 1 (1963) 371-381.

[6] T. Mura, Micromechanics of Defects in Solids, 2nd edition, Martinus Nijhoff, Dordrecht (1987).

[7] H. Bross, Zur Theorie bewegter Versetzungen. phys. stat. sol. 5 (1964) 329-342.

[8] H. Günther, Zur Dynamik schneller Versetzungen. Akademie-Verlag, Berlin (1973).

[9] R.W. Lardner, Mathematical Theory of Dislocations and Fracture, University of Toronto Press, Toronto (1974).

[10] J.D. Eshelby, The force on an elastic singularity. Phil. Trans. Roy. Soc. London A 244 (1951) 87-112.

[11] J.D. Eshelby, The equation of motion of a dislocation. Phys. Rev. 90 (1953) 248-255.

[12] W.S. Wladimirow, Equations of Mathematical Physics, Berlin: Deutscher Verlag der Wissenschaften, 1971 (in German).

[13] F.R.N. Nabarro, The synthesis of elastic dislocation fields. Phil. Mag. 7 (1951) 12241231.

[14] J. Kiusalaas and T. Mura, On the elastic field around an edge dislocation with application to dislocation vibration. Phil. Mag. 9 (1964) 1-7.

[15] J.P. Hirth and J. Lothe, Theory of Dislocations, 2nd edition, John Wiley, New York (1982).

[16] C. Teodosiu, Elastic Models of Crystal Defects, Springer-Verlag, Berlin (1982).

[17] F.R.N. Nabarro, Theory of Crystal Dislocations, Dover, New York (1987).

[18] S. Li and G. Wang, Introduction to Micromechanics and Nanomechanics, World Scientific, Singapore (2008). 
[19] X. Markenscoff, On the dislocation fields in terms of the dynamic Green's function. J. Elasticity 13 (1983) 237-241.

[20] X. Markenscoff and R.J. Clifton, Radiation from expanding circular dislocation loops and elastic precursor decay. ASME Journal of Applied Mechanics 49 (1982) 792-796.

[21] X. Markenscoff and L. Ni, On the effect of dislocation loop curvature on elastic precursor decay. ASME Journal of Applied Mechanics 51 (1984) 753-758.

[22] J. Kiusalaas and T. Mura, On the motion of a screw dislocation, in: Recent Advances in Engineering Science (A. C., Eringen, ed.) Gordon \& Breach, New York (1965) pp. $543-564$.

[23] A.M. Kosevich, Crystal dislocations and the theory of elasticity. in: Dislocations in Solids Vol. 1. F.R.N. Nabarro, ed., North-Holland, Amsterdam (1979) pp. 33-165.

[24] E. Kossecka, Theory of dislocation lines in a continuous medium. Arch. Mech. Stos. 21 (1969) 167-190.

[25] E. Kossecka, Mathematical theory of defects. part II. Dynamics. Arch. Mech. 27 (1975) 79-92.

[26] E. Kossecka and R. deWit, Disclination kinematics. Arch. Mech. 29 (1977) 633-651.

[27] M. Lazar and C. Anastassiadis, The gauge theory of dislocations: conservation and balance laws. Phil. Mag. 88 (2008) 1673-1699.

[28] M. Lazar, On the fundamentals of the three-dimensional translation gauge theory of dislocations, Mathematics and Mechanics of Solids (2011), in press, DOI: $10.1177 / 1081286510370889$.

[29] C. Teodosiu, A dynamic theory of dislocations and its applications to the theory of the elastic-plastic continuum. in Fundamental Aspects of Dislocation Theory, Vol. 2, Eds. J.A. Simmons, R. deWit and R. Bullough, Nat. Bur. Stand. (U.S.), Spec. Publ. 317 (1970) pp. 837-876.

[30] A.C. Eringen and S.S. Suhubi, Elastodynamics, Volume II, Linear Theory. Academic Press, New York (1975).

[31] E. Kausel, Fundamental Solutions in Elastodynamics, Cambridge University Press (2006).

[32] R. deWit, Theory of disclinations IV, J. Res. Nat. Bur. Stand. (U.S.) 77A (1973) 607-658. 